PROCEEDINGS OF THE

AMERICAN MATHEMATICAL SOCIETY

Volume 129, Number 6, Pages 1769-1773

S 0002-9939(00)05804-

Article electronically published on November 3, 2000

\title{
TRANSFORMATION FORMULAS FOR THE BERGMAN KERNELS AND PROJECTIONS OF REINHARDT DOMAINS
}

\author{
SIQI FU
}

(Communicated by Albert Baernstein II)

\begin{abstract}
Formulas that relate the Bergman kernel and projection of a bounded Reinhardt domain whose closure does not intersect the coordinate planes to those of its covering tube domain are obtained via the Poisson summation formula.
\end{abstract}

\section{INTRODUCTION}

It was proved by Steven Bell [2, 3] that the Bergman projections and kernels transform under proper mappings in similar ways as under biholomorphic mappings. These transformation rules have important applications to the study of boundary regularity of proper mappings (see the surveys [1] 5] for details). They are also effective tools in establishing explicit formulas for the Bergman kernels (cf. 4]). Since proper mappings are finite branched covering mappings, it is natural to ask to what extent Bell's formulas can be generalized to infinite covering mappings. One obstacle for such a generalization is the fact that the product of the pull-back of a square-integrable function on the base space and the Jacobian determinant of the covering map is no longer square-integrable on the covering space. In this paper, we study this problem in the case of bounded Reinhardt domains and their covering tube domains using the Poisson summation formula. We establish transformation formulas similar to those under biholomorphic and proper mappings when the closures of the Reinhardt domains do not intersect the coordinate planes (see Theorem 2 below).

\section{The Bergman kernels of tube Domains}

Let $D$ be a domain in $\mathbb{R}^{n}$ and let $T_{D}=\mathbb{R}^{n}+i D$ be the tube domain in $\mathbb{C}^{n}$ with base $D$. It is well known that the Bergman kernel of $T_{D}$ is given by

$$
K_{T_{D}}(z, w)=\frac{1}{(2 \pi)^{n}} \int_{\mathbb{R}^{n}} \frac{e^{i(z-\bar{w}) \cdot t}}{J(t)} d t
$$

where $J(t)=\int_{D} e^{-2 t \cdot y} d y$ (see [7, 6, 8] and references therein). Here we assume that $1 / J(t)=0$ if $J(t)=\infty$. The Bergman space is trivial if and only if $J(t)=\infty$ a.e. on $\mathbb{R}^{n}$.

Received by the editors September 10, 1999 and, in revised form, October 5, 1999.

2000 Mathematics Subject Classification. Primary 32A25, 32H02.

(C)2000 American Mathematical Society 
Let $y \in D$ and assume that $\operatorname{dist}(y, \partial D) \geq d>0$. Then

$$
J(t) \geq \prod_{j=1}^{n} \int_{y_{j}-d / \sqrt{n}}^{y_{j}+d / \sqrt{n}} e^{-2 t_{j} \zeta_{j}} d \zeta_{j}=e^{-2 y \cdot t} \prod_{j=1}^{n} \frac{\sinh \left(2 d t_{j} / \sqrt{n}\right)}{t_{j}} .
$$

Suppose that $v \in D$ with $\operatorname{dist}(v, \partial D) \geq d$. Let $g(y+v, t)=e^{-(y+v) \cdot t} / J(t)$. Applying (2) to both $y$ and $v$, taking the product and square root, we have,

$$
g(y+v, t) \leq \prod_{j=1}^{n} \frac{t_{j}}{\sinh \left(2 d t_{j} / \sqrt{n}\right)}
$$

Therefore,

$$
\int_{\mathbb{R}^{n}} g(y+v, t) d t \leq\left(\frac{n \pi \sqrt{6}}{4}\right)^{n} \frac{1}{d^{2 n}} .
$$

Assume that $D \subset\left\{y \in \mathbb{R}^{n},|y| \leq M\right\}$ for some positive constant $M$. By the definition of $J(t)$,

$$
\left|\frac{\partial^{\alpha}}{\partial t^{\alpha}} J(t)\right| \leq(2 M)^{|\alpha|} J(t)
$$

Therefore

$$
\left|\frac{\partial^{\alpha}}{\partial t^{\alpha}} g(y+v, t)\right| \leq C g(y+v, t)
$$

where $C>0$ is a constant depending only on $\alpha$ and $M$. (In what follows, we will use $C$ to denote constants which could be different in different appearances.) Applying integration by parts to (10) and using (6), we have that

$$
\begin{aligned}
\left|K_{T_{D}}(z, w)\right| & \leq \frac{1}{(2 \pi)^{n}\left|(x-u)^{\alpha}\right|} \int_{\mathbb{R}^{n}}\left|\frac{\partial^{\alpha}}{\partial t^{\alpha}} g(y+v, t)\right| d t \\
& \leq \frac{C}{\left|(x-u)^{\alpha}\right|} \int_{\mathbb{R}^{n}} g(y+v, t) d t,
\end{aligned}
$$

where $z=x+i y$ and $w=u+i v$. Multiplying the denominator of (7) to both sides and using the binomial formula, we then obtain that

$$
\left|K_{T_{D}}(z, w)\right| \leq \frac{C}{\left(1+|x-u|^{2}\right)^{\alpha}} \int_{\mathbb{R}^{n}} g(y+v, t) d t
$$

Let $Q=[0,2 \pi]^{n}$ and $Q_{D}=Q+i D$. Then

$$
\begin{aligned}
\left\|K_{T_{D}}(z+2 k \pi, \cdot)\right\|_{L^{2}\left(Q_{D}\right)} \leq & \left\{\int_{Q} \frac{C}{\left(1+|x+2 k \pi-u|^{2}\right)^{2 \alpha}} d u\right\}^{1 / 2} \\
& \times\left\{\int_{D}\left|\int_{\mathbb{R}^{n}} g(y+v, t) d t\right|^{2} d v\right\}^{1 / 2} .
\end{aligned}
$$

By the Minkowski inequality, the last term above is less than or equal to

$$
\int_{\mathbb{R}^{n}}\left\{\int_{D} g(y+v, t)^{2} d v\right\}^{1 / 2} d t=\int_{\mathbb{R}^{n}} \frac{e^{-y t}}{\sqrt{J(t)}} d t
$$


It follows from (2) that the last integral is finite. Therefore, by choosing $\alpha>n / 4$, we have

$$
\sum_{k \in \mathbb{Z}^{n}}\left\|K_{T_{D}}(z+2 k \pi, \cdot)\right\|_{L^{2}\left(Q_{D}\right)}<\infty .
$$

We summarize what we have obtained so far in the following proposition.

Proposition 1. Let $D$ be a bounded domain in $\mathbb{R}^{n}$ and let $T_{D}=\mathbb{R}^{n}+i D$. Let $z=x+i y$ and $w=u+i v$.

(1) For any $\alpha>0$, there exists a constant $C>0$, depending only on $n, \alpha$, and the diameter of $D$, such that

$$
\left|K_{T_{D}}(z, w)\right| \leq \frac{C}{\left(1+|x-u|^{2}\right)^{\alpha} d^{2 n}},
$$

where $d$ is the minimum of the distances of $y$ and $v$ to $\partial D$.

(2) For any $z \in T_{D}$,

$$
\sum_{k \in \mathbb{Z}^{n}}\left\|K_{T_{D}}(z+2 k \pi, \cdot)\right\|_{L^{2}\left(Q_{D}\right)}<\infty
$$

\section{The Bergman kernels of Reinhardt domains}

Let $\Omega$ be a Reinhardt domain in $\mathbb{C}^{n}$. Let $V_{j}=\left\{\left(z_{1}, \ldots, z_{n}\right) \in \mathbb{C}^{n} ; z_{j}=0\right\}$ and $V=\bigcup_{j=1}^{n} V_{n}$. Let

$$
D=\log |\Omega|=\left\{\left(\log \left|z_{1}\right|, \ldots, \log \left|z_{n}\right|\right) ; \quad\left(z_{1}, \ldots, z_{n}\right) \in \Omega \backslash V\right\} .
$$

Then $T_{D}=\mathbb{R}^{n}+i D$ is a covering of $\Omega \backslash V$ via the maps $\zeta_{j}=e^{-i z_{j}}, 1 \leq j \leq n$. Note that the Bergman kernel of $\Omega \backslash V$ is the same as that of $\Omega$. We will use $P$ to denote the Bergman projection.

Theorem 2. Let $\Omega$ be a bounded Reinhardt domain in $\mathbb{C}^{n}$. Let $D=\log |\Omega|$ and $T_{D}=\mathbb{R}^{n}+i D$. Let $f(z)=\left(e^{-i z_{1}}, \ldots, e^{-i z_{n}}\right)$ and $J f(z)$ be its Jacobian determinant. Suppose that $\operatorname{cl}(\Omega) \cap V=\emptyset$. Then

$$
K_{\Omega}(f(z), f(w))=\frac{1}{J f(z) \overline{J f(w)}} \sum_{k \in \mathbb{Z}^{n}} K_{T_{D}}(z, w+2 k \pi),
$$

where the infinite series converges local uniformly for $z, w \in T_{D}$ and in $L^{2}\left(Q_{D}\right)$ norm for any fixed $z$. Furthermore, for any $\varphi(z) \in L^{2}(\Omega)$,

$$
P_{T_{D}}(J f \cdot(\varphi \circ f))(z)=J f(z) \cdot P_{\Omega}(\varphi)(f(z)) .
$$

It seems that identities (14) and (15) do not follow directly from the methods in 2] and [3. The main reason is that $(\varphi \circ f) \cdot J f$ is no longer in $L^{2}\left(T_{D}\right)$. However, as we shall see later in the proof, $K_{T_{D}}(z, w) \varphi(f(w)) J f(w)$ is integrable on $T_{D}$ for any fixed $z \in T_{D}$. Therefore $P_{T_{D}}(J f \cdot(\varphi \circ f))$ is well defined.

Proof. (1) It is easy to see that

$$
\left\|z^{k}\right\|^{2}=(2 \pi)^{n} \int_{|\Omega|} r^{2 k+\mathbf{1}} d r=(2 \pi)^{n} J(-(k+\mathbf{1})),
$$


where $\mathbf{1}=(1, \ldots, 1)$. Therefore

$$
\begin{aligned}
K_{\Omega}(f(z), f(w)) & =\frac{e^{i(z-\bar{w})}}{(2 \pi)^{n}} \sum_{k \in \mathbb{Z}^{n}} \frac{e^{-i(z-\bar{w}) \cdot(k+\mathbf{1})}}{J(-(k+\mathbf{1}))} \\
& =\frac{e^{i(z-\bar{w})}}{(2 \pi)^{n}} \sum_{k \in \mathbb{Z}^{n}} \frac{e^{-(y+v) \cdot k}}{J(k)} e^{i(x-u) \cdot k} \\
& =\frac{e^{i(z-\bar{w})}}{(2 \pi)^{n}} \sum_{k \in \mathbb{Z}^{n}} g(y+v, k) e^{i(x-u) \cdot k} .
\end{aligned}
$$

Note that under the assumption, $D=\log |\Omega|$ is bounded. The equality (14) then follows from Proposition 1 and the Poisson summation formula (cf. 9], Chapter VII).

(2) It follows from the Schwarz inequality and Proposition 1 (2) that

$$
\sum_{k \in \mathbb{Z}^{n}} \int_{Q_{D}}\left|K_{T_{D}}(z, w+2 k \pi) \varphi(f(w)) J f(w)\right| d V(w)<+\infty,
$$

for any $z \in T_{D}$. Hence $K_{T_{D}}(z, w) \varphi(f(w)) J f(w) \in L^{1}\left(T_{D}\right)$. Therefore the righthand side of (15) equals

$$
\begin{aligned}
J f(z) & \int_{Q_{D}} K_{\Omega}(f(z), f(w)) \varphi(f(w))|J f(w)|^{2} d V(w) \\
& =\int_{Q_{D}} \sum_{k \in \mathbb{Z}^{n}} K_{T_{D}}(z, w+2 k \pi) \varphi(f(w)) J f(w) d V(w) \\
& =\sum_{k \in \mathbb{Z}^{n}} \int_{Q_{D}} K_{T_{D}}(z, w+2 k \pi) \varphi(f(w)) J f(w) d V(w) \\
& =\int_{T_{D}} K_{T_{D}}(z, w) \varphi(f(w)) J f(w) d V(w) .
\end{aligned}
$$

The exchange of the integral and the summation signs is justified by (16). We thus establish (15).

Remark. In the case when $\Omega$ is the unit disc and $T_{D}$ is the lower half-plane, the identity (14) is the same as the following well-known identity:

$$
\frac{\pi^{2}}{\sin ^{2}(\pi z)}=\sum_{\alpha=-\infty}^{\infty} \frac{1}{(z-\alpha)^{2}} .
$$

It would be desirable to establish (14) and (15) for all bounded Reinhardt domains and to generalize Bell's transformation formulas to other covering spaces.

\section{ACKNOWLEDGMENT}

The author is indebted to Professor Peter Polyakov for many constructive discussions and suggestions. Part of this work was done while the author was a postdoc at Texas A \& M University in 1996-98. The author thanks Professors Harold Boas and Emil Straube for stimulating conversations and continuing support. 


\section{REFERENCES}

[1] Eric Bedford, Proper holomorphic mappings, Bull. Amer. Math. Soc. (NS) 10 (1984), no. 2, 157-175. MR 85b:32041

[2] Steven R. Bell, Proper holomorphic mappings and the Bergman projection, Duke Math. Jour. 48 (1981), no. 1, 167-175. MR 82d:32011

[3] - The Bergman kernel function and proper holomorphic mappings, Trans. Amer. Math. Soc. 270 (1982), no. 2, 685-691. MR 83i:32033

[4] Harold P. Boas, Siqi Fu, and Emil J. Straube, The Bergman kernel function: explicit formulas and zeroes, Proc. Amer. Math. Soc. 127 (1998), no. 3, 805-811. MR 99f:32037

[5] Franc Forstnerič, Proper holomorphic mappings: a survey, Several Complex Variables: Proceedings of the Mittag-Leffler Institute, 1987-1988, edited by John Erik Fornæss, Princeton University Press, 1993, pp. 297-363. MR 94a:32042

[6] T. G. Genchev, Paley-Wiener type theorems for functions in Bergman spaces over tube domains, Jour. of Math. Anal. and Appl. 118 (1986), 496-501. MR 87k:32003

[7] Adam Korányi, The Bergman kernel function for tubes over convex cones, Pacific Jour. of Math. 12 (1962), 1355-1359. MR 27:1623

[8] Saburou Saitoh, Fourier-Laplace transforms and the Bergman spaces, Proc. Amer. Math. Soc. 102 (1988), no. 4, 985-992. MR 89c:32014

[9] Elias M. Stein and Guido Weiss, Introduction to Fourier analysis on Euclidean spaces, Princeton University Press, 1971. MR 46:4102

Department of Mathematics, University of Wyoming, Laramie, Wyoming 82071

E-mail address: sfu@uwyo.edu 